\title{
Randomization inference with general interference and censoring
}

\author{
Wen Wei Loh $^{1}$ | Michael G. Hudgens ${ }^{2} \mid$ John D. Clemens ${ }^{3} \mid$ Mohammad Ali $^{4} \mid$ \\ Michael E. Emch ${ }^{5}$
}

${ }^{1}$ Department of Data Analysis, Ghent

University, Gent, Belgium

${ }^{2}$ Department of Biostatistics, University of North Carolina, Chapel Hill, North

Carolina

${ }^{3}$ Department of Epidemiology, University

of California, Los Angeles, California

${ }^{4}$ Department of International Health,

Johns Hopkins University, Baltimore,

Maryland

${ }^{5}$ Department of Geography, University of

North Carolina, Chapel Hill, North

Carolina

Correspondence

Wen Wei Loh, Department of Data

Analysis, Ghent University, Henri

Dunantlaan 1, Gent 9000, Belgium. Email:

WenWei.Loh@UGent.be

Funding information

National Institutes of Health, Grant/

Award Number: R01 AI085073

\begin{abstract}
Interference occurs between individuals when the treatment (or exposure) of one individual affects the outcome of another individual. Previous work on causal inference methods in the presence of interference has focused on the setting where it is a priori assumed that there is "partial interference," in the sense that individuals can be partitioned into groups wherein there is no interference between individuals in different groups. Bowers et al. (2012, Political Anal, 21, 97-124) and Bowers et al. (2016, Political Anal, 24, 395-403) consider randomization-based inferential methods that allow for more general interference structures in the context of randomized experiments. In this paper, extensions of Bowers et al. that allow for failure time outcomes subject to right censoring are proposed. Permitting right-censored outcomes is challenging because standard randomization-based tests of the null hypothesis of no treatment effect assume that whether an individual is censored does not depend on treatment. The proposed extension of Bowers et al. to allow for censoring entails adapting the method of Wang et al. (2010, Biostatistics, 11, 676-692) for two-sample survival comparisons in the presence of unequal censoring. The methods are examined via simulation studies and utilized to assess the effects of cholera vaccination in an individually randomized trial of 73000 children and women in Matlab, Bangladesh.
\end{abstract}

K E Y W O R D S

causal inference, censoring, interference, permutation test, randomization inference, spillover effects

\section{1 | INTRODUCTION}

Interference arises when an individual's potential outcomes depend on the treatment status of others. Assuming interference is absent when assessing the causal effect of a treatment on an outcome may be scientifically implausible in certain settings. For example, in the study of infectious diseases, whether one individual receives a vaccine may affect whether another individual becomes infected or develops the disease. Motivated by infectious diseases and other settings where individuals interact, many existing causal inference methods have been extended to allow for interference, see Halloran and Hudgens (2016) for a recent review.

Some previous work on causal inference methods in the presence of interference has assumed a priori that there is partial interference (Sobel, 2006), that is, individuals can be partitioned into groups wherein there is no interference between individuals in different groups. In this paper, we consider the more general setting where interference between any two individuals may be assumed. Recent approaches that allow for the presence of general interference 
when evaluating treatment effects include Bowers et al. (2012; 2016), Sussman and Airoldi (2017), and Athey et al. (2018) among others. In randomized experiments where the treatment assignment mechanism is known, Bowers et al. (2012) (henceforth BFP) described how to carry out randomization-based (ie, permutation or design-based) inference on parameters in causal models, which allow for general interference. For an assumed causal model, a randomization-based approach entails constructing confidence sets for the causal parameters by inverting a set of hypothesis tests. An appealing aspect of randomizationbased inference (Rosenbaum, 2002, chapter 2) is that no assumption of random sampling from some hypothetical superpopulation is invoked. Another benefit is that the resulting $100(1-\alpha) \%$ confidence sets are exact, that is, the probability the true causal parameters are contained in a confidence set is at least the nominal level $1-\alpha$. Moreover, in settings where possible interference is a priori assumed to have a specified network structure, it is unreasonable to assume that individual outcomes are independent, such that standard frequentist approaches are not justified; in contrast, randomization-based methods that allow for possible general interference readily apply.

In this article, we propose extensions of Bowers et al. to the setting where the response of interest is a failure time, and only the censoring time is observed for a subset of individuals due to right censoring. In general, when there is right censoring, randomization-based inference on the failure times is exact only when treatment does not affect the censoring times. The proposal to permit right-censored observations thus entails adapting the method of Wang et al. (2010) for two-sample survival comparisons in the presence of unequal censoring. The remainder of this article is as follows. In Section 2, notation is introduced, causal models are defined, and the randomization inferential procedure by Bowers et al. when there is no censoring is reviewed. In Section 3, the proposed extension allowing for right-censored outcomes is presented, and simulation study results are shown demonstrating the method approximately preserves the nominal size over a range of settings. In Section 4, the methods are utilized to assess the effects of cholera vaccination in an individually randomized trial of $n=73000$ women and children in Matlab, Bangladesh. A brief discussion is provided in Section 5 .

\section{2 | GENERAL INTERFERENCE AND CAUSAL MODELS}

\section{1 | General interference}

Consider a finite population of $n$ individuals randomly assigned to either treatment or control. For each individual $i=1, \ldots, n$, let $Z_{i}=1$ if individual $i$ is assigned treatment and $Z_{i}=0$ otherwise. The vector comprising all treatment assignments is denoted as $\mathbf{Z}=\left(Z_{1}, \ldots, Z_{n}\right)$. The uppercase $\mathbf{Z}$ denotes the random variable corresponding to treatment assignment and the lowercase $\mathbf{z}$ denotes the possible realizations of $\mathbf{Z}$. Let $y_{i}(\mathbf{z})$ denote the potential outcome for individual $i$ that would be observed for treatment assignment $\mathbf{z}$; the observed outcome is denoted by $Y_{i}=y_{i}(\mathbf{Z})$. Let $\mathbf{y}(\mathbf{z})=\left(y_{1}(\mathbf{z}), \ldots, y_{n}(\mathbf{z})\right)$ denote the vector of potential outcomes. The potential outcomes $\mathbf{y}(\mathbf{z})$ and $\mathbf{z} \in\{0,1\}^{n}$ are considered fixed features of the finite population of $n$ individuals.

Define the $n \times n$ interference matrix $\mathbb{A}$ with $(i, j)$ entry $A_{i j}$ for $i, j \in\{1, \ldots, n\}$ as follows. Let $A_{i j}=0$ for $i=j$. For $i \neq j$ let $A_{i j}=0$ if it is assumed a priori that individual $j$ does not interfere with individual $i$; otherwise let $A_{i j}=1$. Note that $A_{i j}=0$ implies it is assumed a priori $y_{i}(\mathbf{z})$ does not depend on $z_{j}$, whereas $A_{i j}=1$ merely indicates the possibility that individual $j$ may interfere with individual $i$, and does not necessarily imply $y_{i}(\mathbf{z})$ depends on $z_{j}$. Indeed, one of our primary inferential goals is to determine whether such possible interference is present. The definition of $\mathbb{A}$ encodes the assumption that any spillover effects on individual $i$ may emanate only from individuals $j$ where $A_{i j}=1$, and not from those where $A_{i j}=0$. The exact relationship between $y_{i}(\mathbf{z})$ and $\mathbf{z}$ is specified using a causal model described in the next section. Let the interference set (ie, neighbors) for individual $i$ be the set of individuals $j \in\{1, \ldots, n\} \backslash i$ where $A_{i j}=1$. Denote the $i$ th row of $\mathbb{A}$ by the vector $\mathbf{A}_{i}$, and the size of the interference set by the scalar $A_{i}=\sum_{j=1}^{n} A_{i j}$. Under partial interference, individuals can be partitioned into groups or clusters wherein there is no interference between groups, in which case $\mathbb{A}$ can be expressed as a block-diagonal matrix with each block corresponding to a group. Under general interference, each individual is allowed to have their own possibly unique interference set, so that there is no restriction on the structure of $\mathbb{A}$. Here and throughout $\mathbb{A}$ is assumed known and invariant to treatment.

\subsection{Causal models}

A (counterfactual) causal model expresses the potential outcomes $y_{i}(\mathbf{z})$ as a parametric deterministic function of any treatment $\mathbf{z}$. Following Bowers et al., we consider a class of causal models that entails the composition of two functions. In particular, assume $y_{i}(\mathbf{z})=h\left\{y_{i}(\mathbf{0}) \mid \mathcal{F}(\mathbf{z} ; \theta, \mathbb{A})\right\}$ for user-specified functions $h$ and $\mathcal{F}$, with $y_{i}(\mathbf{0})$ denoting the potential outcome under the uniformity trial (Rosenbaum, 2007) where no one receives treatment. The function $\mathcal{F}(\mathbf{z} ; \theta, \mathbb{A})$ takes as its arguments the treatment vector $\mathbf{z}$, causal parameter $\theta$, and interference 
matrix $\mathbb{A}$. The dependence of $\mathcal{F}(\mathbf{z} ; \theta, \mathbb{A})$ on $i$ is left implicit notationally as it is implied under the specified causal

model. For notational simplicity, we write $\mathcal{F}=\mathcal{F}(\mathbf{z} ; \theta)$,

with the dependence on Aimplicit. The specification of $\mathcal{F}$ determines how an individual's potential outcomes differ across different treatments $\mathbf{z}$ and different values of the parameter $\theta$, and includes, but is not limited to, how direct and spillover effects propagate. The link function $h$ is a oneto-one function mapping $y_{i}(\mathbf{0})$ to $y_{i}(\mathbf{z})$ for a specified $\mathcal{F}$; in particular, the uniformity trial potential outcomes can be determined from the observed data under a specified causal model by $y_{i}(\mathbf{0})=h^{-1}\left\{Y_{i} \mid \mathcal{F}(\mathbf{Z} ; \theta)\right\}$, where $h^{-1}$ is the inverse of $h(a \mid b)$.

In practice, prior beliefs or background knowledge may be used to inform the choice of $\mathcal{F}$ and $h$. We consider two specific causal models, defined in (1) and (2) below, and assume $h(a \mid b)=a \exp (b)$, although the proposed methods are general and apply to other forms of $\mathcal{F}$ and $h$. Denote the number and proportion of individual $i$ 's neighbors assigned to treatment by $T_{i}=\mathbf{A}_{i} \mathbf{Z}^{T}$ and $G_{i}=T_{i} / A_{i}$ respectively; here $A_{i}=0$ implies $T_{i}=G_{i}=0$. Note that $T_{i}$ and $G_{i}$ depend on $\mathbf{Z}$, but this dependence is suppressed for notational convenience. Let

$$
\begin{aligned}
& \mathcal{F}_{\text {add }}(\mathbf{Z} ; \delta, \tau)=\delta Z_{i}+\tau G_{i}, \\
& \mathcal{F}_{\mathrm{BFP}}(\mathbf{Z} ; \delta, \tau)= \delta+\log \left[1+\left(1-Z_{i}\right)\{\exp (-\delta)-1\}\right. \\
&\left.\times \exp \left(-\tau^{2} T_{i}\right)\right] .
\end{aligned}
$$

Under both causal models, the effect of treatment $\mathbf{Z}$ on the outcome for individual $i$ takes the form of a bivariate treatment: $Z_{i}$ is the (individual) treatment received, and $G_{i}$ (or $T_{i}$ ) is the proportion (or number) of individuals in the interference set treated. The parameters $\delta$ and $\tau$ measure the extent to which the potential outcomes increase or decrease, relative to $y_{i}(\mathbf{0})$, due to $Z_{i}$ and $G_{i}$ (or $T_{i}$ ). Causal model (2) was proposed by BFP and restricts interference to those who did not receive treatment, with the direct (or individual) effect parametrized to be larger in magnitude than the spillover (or peer) effect. As both $G_{i}$ and $T_{i}$ depend only on the total number in the interference set treated, a peer effect homogeneity assumption is implied by these two causal models; Hudgens and Halloran (2008) refer to the assumption as stratified interference. Causal models allowing for interference that does not occur via the summary $T_{i}$ can also be utilized within this framework. For example, we might posit $\mathcal{F}=\delta Z_{i}+\tau Z_{M_{i}}$ where $M_{i}=\arg \max _{j: A_{i j}=1} A_{j}$ denotes the neighbor of individual $i$ having the biggest interference set. See Ogburn et al. (2017) and Sussman and Airoldi (2017) for other causal models that allow for interference. The next section describes how to carry out randomization inference for the parameter $\theta=(\delta, \tau)$ under a specified $\mathcal{F}$.

\subsection{Randomization inference}

For a specified causal model $\mathcal{F}$, the uniformity trial potential outcomes under a null hypothesis $H_{0}: \theta=\theta_{0}$ can be determined from the observed data by $y_{i}(\mathbf{0})=Y_{i} \exp$ $\left\{-\mathcal{F}\left(\mathbf{Z} ; \theta_{0}\right)\right\}$. In a randomized experiment where individuals are assigned treatment with equal probability, the uniformity trial outcomes should be similarly distributed between treatment $(Z=1)$ and control $(Z=0)$ groups (Rosenbaum, 2002) if $H_{0}$ is true and $\mathcal{F}$ is correctly specified. Therefore the null hypothesis $H_{0}$ can be tested using a test statistic $\mathcal{T S}\left(\mathbf{Z} ; \theta_{0}\right)$ that compares the uniformity outcomes between treated and untreated individuals. For example, BFP used the two-sample Kolmogorov-Smirnov (KS) test statistic to compare the empirical distributions of the uniformity outcomes in the treatment and control groups. Bowers et al. (2016) proposed a multiple linear regression model of the uniformity outcomes on $Z$ and $T$, using the resulting sum of squares of residuals as a test statistic.

For a chosen test statistic $\mathcal{T S}\left(\mathbf{Z} ; \theta_{0}\right)$, the plausibility of $H_{0}$ can be assessed by evaluating the frequency of obtaining a value at least as "extreme" (from $H_{0}$ ) as the observed value, over hypothetical reassignments of $\mathbf{Z}$ under $H_{0}$. Here and throughout a completely randomized experiment is assumed, where the number assigned to treatment, denoted by $m=\sum_{i=1}^{n} Z_{i}$, is fixed by design. The sample space of all hypothetical reassignments $\mathbf{Z}$ is the set of vectors of length $n$ containing $m$ 1's and $n-m \quad 0$ 's, and is denoted by $\Omega=\left\{\mathbf{z}: z_{i} \in\{0,1\}, i=1, \ldots, n, \sum_{i=1}^{n} z_{i}=m\right\}$. Each reassignment occurs with probability $|\Omega|^{-1}$, so that a two-sided $p$ value may be defined as $\operatorname{pv}\left(\theta_{0}\right)=|\Omega|^{-1}$ $\sum_{\mathbf{z} \in \Omega} I\left\{\mathcal{T S}\left(\mathbf{z} ; \theta_{0}\right) \geqslant \mathcal{T S}\left(\mathbf{Z} ; \theta_{0}\right)\right\}$, where, without loss of generality, it is assumed that the larger values of $\mathcal{T S}\left(\mathbf{Z} ; \theta_{0}\right)$ suggest stronger evidence against $H_{0}$, and $I\{B\}=1$ if $B$ is true and 0 otherwise. When it is not computationally feasible to enumerate $\Omega$ exactly, an approximation of $\Omega$ based on $C$ random draws of $\mathbf{z}$ from $\Omega$ may be used to yield an approximate $p$ value, denoted by $\operatorname{pv}^{C}\left(\theta_{0}\right)$.

Confidence sets can be constructed by test inversion. The subset of $\theta_{0}$ values where $\operatorname{pv}\left(\theta_{0}\right)$, or $\mathrm{pv}^{C}\left(\theta_{0}\right)$, is greater than or equal to $\alpha$ forms a $100(1-\alpha) \%$ exact confidence set for $\theta$. Confidence sets for individual parameters in $\theta$ can be obtained readily from a confidence set for $\theta$. For example, a $100(1-\alpha) \%$ confidence set for $\delta$ is given by all values of $\delta_{0}$ such that there exists some value of $\tau_{0}$ where $\left(\delta_{0}, \tau_{0}\right)$ is in the $100(1-\alpha) \%$ confidence set for $(\delta, \tau)$.

It is important to note that each hypothesis test assesses the compatibility of the observed data with the assumed 
causal model $\mathcal{F}$ and assumed parameter values $\theta_{0}$ specified by $\mathcal{F}$ under the null. Rejection of the hypothesis only indicates that either $\mathcal{F}$ or $\theta_{0}$ is implausible. In some circumstances, all feasible parameter values for an assumed causal model may be rejected, leading to an empty confidence set. This indicates that all possible parameter values are implausible, implying that the assumed causal model provides a poor fit to the data.

\section{3 | RIGHT-CENSORED FAILURE TIME OUTCOMES}

Now suppose each individual's outcome is a (positive) failure time, subject to right censoring if the individual is not followed long enough for failure to be observed. For $i=1, \ldots, n$, let $\tilde{Y}_{i}$ and $C_{i}$ denote the failure time and the censoring time, respectively. The failure time $\tilde{Y}_{i}$ is observed only if $\tilde{Y}_{i} \leqslant C_{i}$, so that the observed data are $Y_{i}=\min \left\{\tilde{Y}_{i}, C_{i}\right\}$ and the failure indicator $D_{i}=I\left\{\tilde{Y}_{i} \leqslant C_{i}\right\}$. The outcomes being right-censored causes two complications for the randomization inference approach described in Section 2. First, the test statistic employed needs to account for right censoring; some possible statistics are discussed in Section 3.1. Second, the null hypothesis $H_{0}: \theta=\theta_{0}$ for a specified causal model $\mathcal{F}$ is no longer sharp in the sense that not all uniformity trial potential outcomes can be determined from the observed data under $H_{0}$. To see this, define $y_{i}(\mathbf{0})=Y_{i} \exp \left\{-\mathcal{F}\left(\mathbf{Z} ; \theta_{0}\right)\right\}$, which can be determined from the observed data as in the previous section. Let $\tilde{y}_{i}(\mathbf{0})=\tilde{Y}_{i} \exp \left\{-\mathcal{F}\left(\mathbf{Z} ; \theta_{0}\right)\right\}$ denote the uniformity trial potential failure time for individual $i$ under $H_{0}$. For individuals who are not censored, $Y_{i}=\tilde{Y}_{i}$ implies $y_{i}(\mathbf{0})=\tilde{y}_{i}(\mathbf{0})$, that is, the uniformity trial potential failure time can be determined exactly under $H_{0}$ if $D_{i}=1$. But for individuals who are censored, $\tilde{Y}_{i}$ is unobserved, so that $\tilde{y}_{i}(\mathbf{0})$ is unknown under $H_{0}$. Nonetheless, it is known for these individuals that $Y_{i}<\tilde{Y}_{i}$; multiplying both sides of this inequality by $\exp \left\{-\mathcal{F}\left(\mathbf{Z} ; \theta_{0}\right)\right\}$, it follows that $y_{i}(\mathbf{0})<\tilde{y}_{i}(\mathbf{0})$. Thus the observed censoring times provide some information about the unknown failure times $\tilde{y}_{i}(\mathbf{0})$ for rightcensored individuals. In particular, $y_{i}(\mathbf{0})$ serves as a lower bound for $\tilde{y}_{i}(\mathbf{0})$ under $H_{0}$. Because the null hypothesis is no longer sharp, the randomization testing approach in Section 2.3 in the absence of censoring requires modification; the proposed approach is described in Section 3.2.

\section{1 | Test statistics that accommodate right censoring}

The test statistics considered in Section 2.3 require modification to accommodate right censoring. Instead of the KS statistic, the log-rank (LogR) statistic may be used to compare the right-censored uniformity failure times in the treatment and control groups. An analog of the multiple linear regression model is the parametric accelerated failure time (AFT) model where the logtransformed failure times are linear functions of the predictors. In the following, we consider a log-normal AFT model of the uniformity failure times given by $\log \tilde{y}_{i}(\mathbf{0})=\mathbf{q}_{i} \beta+\sigma \epsilon_{i}$, where $\mathbf{q}_{i}=\left(1, Z_{i}, G_{i}, Z_{i} G_{i}, A_{i}\right)$ and the errors $\epsilon_{i}$ are independent and normally distributed with mean zero and variance one. (For the $\mathcal{F}_{\mathrm{BFP}}$ causal model, $G_{i}$ may be replaced by $T_{i}$.) Following the likelihood ratio principle for testing, a likelihood ratio permutation test is expected to be the most powerful test against certain alternatives (see Lehmann and Romano, 2005, chapter 5.9 for an example in the setting where there is no interference and no censoring). Let $\mathbf{D}=\left(D_{1}, \ldots, D_{n}\right)$ denote the vector of failure indicators, and denote the log-likelihood by

$$
\begin{aligned}
l\left(\mathbf{Z}, \mathbf{D} ; \boldsymbol{\beta}, \sigma, \theta_{0}\right)= & \sum_{i=1}^{n}\left[D_{i} \log \left\{\boldsymbol{\phi}\left(\epsilon_{i}\right) /\left(\sigma \tilde{y}_{i}(\mathbf{0})\right)\right\}\right. \\
& \left.+\left(1-D_{i}\right) \log \left\{1-\Phi\left(\epsilon_{i}\right)\right\}\right],
\end{aligned}
$$

where $\epsilon_{i}=\left\{\log \tilde{y}_{i}(\mathbf{0})-\mathbf{q}_{i} \beta\right\} / \sigma$, and $\phi$ and $\Phi$ are the standard normal density and distribution functions, respectively; see, for example, equation (6.25) of Collett (2003). Let $\hat{\beta}$ and $\hat{\sigma}$ denote the maximum likelihood estimates (MLEs), and let $\tilde{\beta}$ and $\tilde{\sigma}$ denote the MLEs for the "intercept-only" model, that is, under the restriction $\beta=(1,0,0,0,0)^{T}$. Then the log-likelihood difference is $\operatorname{LRaft}\left(\mathbf{Z}, \mathbf{D} ; \theta_{0}\right)=l\left(\mathbf{Z}, \mathbf{D} ; \hat{\boldsymbol{\beta}}, \hat{\sigma}, \theta_{0}\right)-l\left(\mathbf{Z}, \mathbf{D} ; \tilde{\boldsymbol{\beta}}, \tilde{\sigma}, \theta_{0}\right)$. In practice, $l\left(\mathbf{Z}, \mathbf{D} ; \hat{\boldsymbol{\beta}}, \hat{\sigma}, \theta_{0}\right)$ can be used in place of $\operatorname{LRaft}\left(\mathbf{Z}, \mathbf{D} ; \theta_{0}\right)$ since $l\left(\mathbf{Z}, \mathbf{D} ; \tilde{\boldsymbol{\beta}}, \tilde{\sigma}, \theta_{0}\right)$ is constant with respect to $\mathbf{Z}$ for a fixed value $\theta_{0}$. Note the AFT model should only be considered as a "working model," used solely to generate a test statistic for a hypothesis testing procedure. Under the randomization-based framework, valid inference does not rely on this working model being correctly specified. Rather, $l\left(\mathbf{Z}, \mathbf{D} ; \hat{\boldsymbol{\beta}}, \hat{\sigma}, \theta_{0}\right)$ can simply be viewed as a mathematical (scalar) summary of $\{\mathbf{y}(\mathbf{0}), \mathbf{D}, \mathbf{Z}\}$ that is compared against other treatment assignments for assessing the plausibility of $H_{0}: \theta=\theta_{0}$.

\section{2 | Correcting for right-censored uniformity trial failure times}

The randomization-based inferential procedures described in Section 2.3 do not necessarily yield tests that preserve the nominal size in the presence of right censoring, even if the test statistics considered in Section 3.1 are utilized. Randomization tests of no treatment effect on the failure times in the presence of censoring 
generally only preserve the nominal size when treatment does not affect the censoring times. To see this, consider for a moment the setting where there is no interference between individuals, so that each individual has two potential failure time outcomes $\tilde{y}_{i}(0)$ and $\tilde{y}_{i}(1)$, and two potential censoring times $c_{i}(0)$ and $c_{i}(1)$. Let $\tilde{Y}_{i}=\tilde{y}_{i}\left(Z_{i}\right)$ and $C_{i}=c_{i}\left(Z_{i}\right)$, and define $Y_{i}$ and $D_{i}$ as above. Consider testing the null hypothesis of no individual-level treatment effect, that is, $H_{0}: \tilde{y}_{i}(0)=\tilde{y}_{i}(1)$ for $i=1, \ldots, n$, using some test statistic which is a function of $\{\mathbf{Y}, \mathbf{D}, \mathbf{Z}\}$ where $\mathbf{Y}=\left(Y_{1}, \ldots, Y_{n}\right)$ is the vector of observed outcomes. If we assume $c_{i}(0)=c_{i}(1)$, then under the null, both $Y_{i}$ and $D_{i}$ will be the same regardless of treatment, allowing exact determination of the test statistic's sampling distribution by enumeration over all possible reassignments in $\Omega$.

However, when inverting a randomization test to construct a confidence set, null hypotheses corresponding to nonzero treatment effects on the failure times must also be tested. For such null hypotheses, the standard randomization testing approach described in Section 2.3 cannot be used to determine a test statistic's sampling distribution under the null, because in general, an individual's censoring indicator $D_{i}$ will not be fixed over all possible reassignments $\mathbf{z} \in \Omega$, even if treatment has no effect on the censoring times. To see this, returning to the setting where there is interference consider the causal model $\mathcal{F}_{\text {add }}$ and suppose $Z_{i}=1$ and $D_{i}=0$, that is, individual $i$ is assigned treatment and is censored at time $Y_{i}$ with failure time $\tilde{Y}_{i}>Y_{i}$. Further assume treatment has no effect on the censoring times, so that the potential censoring time for individual $i$ equals $Y_{i}$ for all treatments $\mathbf{z} \in \Omega$. Now consider testing $H_{0}: \theta=\theta_{0}$ where $\delta_{0}>\log \left(\tilde{Y}_{i} / Y_{i}\right)$ and $\tau_{0}=0$. Then for treatment reassignment $\mathbf{z}^{\prime} \in \Omega$ where $z_{i}^{\prime}=0$ it follows that $\tilde{y}_{i}\left(\mathbf{z}^{\prime}\right)=\tilde{y}_{i}(\mathbf{0})=\tilde{Y}_{i} \exp \left(-\delta_{0}\right)<Y_{i}$, that is, individual $i$ would not be censored for treatment $\mathbf{z}^{\prime}$. Thus, as will be demonstrated empirically in Section 3.3 below, a randomization test that holds the set of censored individuals fixed over treatment reassignments will not in general control the type I error. Instead, we propose the following randomization-based inferential procedure that allows the set of censored individuals to vary over reassignments.

The procedure entails adapting the $I P_{Z}$ permutation test by Wang et al. (2010). An outline of the procedure is as follows. First, $\mathbf{y}(\mathbf{0})$ is determined under $H_{0}$ using the specified causal model $\mathcal{F}$ and a test statistic from Section 3.1 is evaluated at $\{\mathbf{y}(\mathbf{0}), \mathbf{D}, \mathbf{Z}\}$. Second, the sampling distribution of the test statistic under $H_{0}$ over hypothetical treatment reassignments is approximated by: (a) imputing the unknown uniformity trial failure times for censored individuals according to the assumed causal model $\mathcal{F}$ under $H_{0}$ and (b) nonparametrically imputing censoring times using treatment group-specific Kaplan-Meier (KM) estimators of the censoring time distributions. No causal model is assumed for the censoring times.

The specific procedure is as follows. For a single observed dataset $\{\mathbf{Y}, \mathbf{D}, \mathbf{Z}\}$, the following steps are carried out to test $H_{0}:(\delta, \tau)=\left(\delta_{0}, \tau_{0}\right)$ :

1. Determine the possibly right-censored uniformity trial potential failure times under $H_{0}$, for example, under the causal model $\mathcal{F}_{\text {add }}, y_{i}(\mathbf{0})=Y_{i} \exp \left\{-\left(\delta_{0} Z_{i}+\tau_{0} G_{i}\right)\right\}$. Calculate the observed value of the chosen test statistic, for example, the log-rank statistic, using $\{\mathbf{y}(\mathbf{0}), \mathbf{D}, \mathbf{Z}\}$.

(a) Compute the KM estimator of the distribution function of the uniformity failure times under $H_{0}$ using $\{\mathbf{y}(\mathbf{0}), \mathbf{D}\}$. Denote the estimator by $\hat{F}_{0}(\cdot)$.

(b) For $z=0,1$, among individuals with treatment $Z_{i}=z$, compute the group-specific KM estimator of the censoring time distribution, using the observed times $Y_{i}$ and censoring indicators $1-D_{i}$. Denote the estimators by $\hat{S}(\cdot \mid z)$.

2. Randomly sample a new treatment assignment $\mathbf{z} \in \Omega$.

3. If $D_{i}=1$, set $\tilde{y}_{i}^{*}(\mathbf{0})=y_{i}(\mathbf{0})$, where $y_{i}(\mathbf{0})=\tilde{y}_{i}(\mathbf{0})$ is the observed uniformity failure time under $H_{0}$. Otherwise if $D_{i}=0$, since $\tilde{y}_{i}(\mathbf{0})$ is unknown, sample a failure time from a truncated distribution with lower bound $y_{i}(\mathbf{0})$ as follows. Randomly draw $u \sim \operatorname{Uniform}\left[\hat{F}_{0}\left(y_{i}(\mathbf{0})\right), 1\right]$. If $u \leqslant \hat{F}_{0}\left(\tilde{y}_{\max }(\mathbf{0})\right)$, where $\tilde{y}_{\max }(\mathbf{0})=\max _{i: D_{i}=1} \tilde{y}_{i}(\mathbf{0})$ is the maximum observed uniformity failure time, set the failure time as $\tilde{y}_{i}^{*}(\mathbf{0})=\hat{F}_{0}^{-1}(u)$; otherwise set $\tilde{y}_{i}^{*}(\mathbf{0})=\tilde{y}_{\max }(\mathbf{0})$. (The ${ }^{*}$ symbol distinguishes the imputed failure times from the unknown failure times $\tilde{y}_{i}(\mathbf{0})$ for those with $D_{i}=0$.) Determine the potential failure times under treatment $\mathbf{z}$ using the assumed causal model and the null parameter values, for example, $\tilde{y}_{i}(\mathbf{z})=\tilde{y}_{i}^{*}(\mathbf{0}) \exp \left(\delta_{0} z_{i}+\tau_{0} g_{i}\right)$, where $g_{i}$ is the realization of $G_{i}$ under treatment $\mathbf{z}$.

4. Sample a censoring time under treatment assignment $\mathbf{z}$, denoted by $c_{i}(\mathbf{z})$, from $\hat{S}\left(\cdot \mid z_{i}\right)$ as follows. Randomly draw $v \sim \operatorname{Uniform}(0,1)$. Let $Y_{\max }=\max _{i: Z_{i}=z_{i}} Y_{i}$ be the maximum observed time among individuals with treatment $Z_{i}=z_{i}$. If $Y_{\max }$ is a censoring time, then the $\mathrm{KM}$ estimator of the censoring time distribution evaluated at $Y_{\max }$ is $\hat{S}\left(Y_{\max } \mid z_{i}\right)=1$. Hence set the censoring time to be $c_{i}(\mathbf{z})=\hat{S}^{-1}\left(v \mid z_{i}\right)$. Otherwise, if $Y_{\max }$ is a failure time so that $\hat{S}\left(Y_{\max } \mid z_{i}\right)<1$, set the censoring time to be $c_{i}(\mathbf{z})=\hat{S}^{-1}\left(v \mid z_{i}\right)$ if $v \leqslant \hat{S}\left(Y_{\max } \mid z_{i}\right)$ and let $c_{i}(\mathbf{z})=Y_{\max }$ otherwise. Hence $c_{i}(\mathbf{z}) \leqslant Y_{\max }$ so that any imputed potential failure time longer than $Y_{\max }$ will be censored at $Y_{\max }$. 
5. Determine the potential outcomes under treatment $\mathbf{z}$ as $y_{i}(\mathbf{z})=\min \left\{\tilde{y}_{i}(\mathbf{z}), c_{i}(\mathbf{z})\right\}$ and the failure indicators as $d_{i}(\mathbf{z})=1$ if $\tilde{y}_{i}(\mathbf{z}) \leqslant c_{i}(\mathbf{z})$ or 0 otherwise.

6. Determine the uniformity outcomes under treatment $\mathbf{z}$ using the same causal model as in step 1, for example, $y_{i}^{\dagger}(\mathbf{0})=y_{i}(\mathbf{z}) \exp \left\{-\left(\delta_{0} z_{i}+\tau_{0} g_{i}\right)\right\}$. (The $\dagger$ symbol denotes the uniformity outcomes determined using $y_{i}(\mathbf{z})$, which differ from the uniformity outcomes determined using $Y_{i}$ in step 1.) Compute the chosen test statistic using $\left\{\mathbf{y}^{\dagger}(\mathbf{0}), \mathbf{d}(\mathbf{z}), \mathbf{z}\right\}$, where $\mathbf{y}^{\dagger}(\mathbf{0})=\left(y_{1}^{\dagger}(\mathbf{0}), \ldots, y_{n}^{\dagger}(\mathbf{0})\right)$ and $\mathbf{d}(\mathbf{z})=\left(d_{1}(\mathbf{z}), \ldots, d_{n}(\mathbf{z})\right)$ are vectors of length $n$.

7. The sampling distribution of the chosen test statistic can be obtained by repeating steps 2 to 6 . The $p$ value for testing $H_{0}$ can be determined by comparing the resulting sampling distribution with the observed value of the chosen test statistic from step 1.

\subsection{Empirical evaluation of proposed tests}

In this section, the ability of the proposed procedure to better control the type I error in the presence of right censoring is assessed empirically. A simulation study is conducted as follows. The total number of individuals $n$ is set to 128 , with exactly $m$ individuals assigned to treatment as in a completely randomized experiment. For each individual $i$, the interference set $\mathbf{A}_{i}$ is generated once as follows: (a) randomly draw the interference set size as $A_{i} \sim$ Poisson(16); (b) sample without replacement $A_{i}$ values of $j \in\{1, \ldots, n\} \backslash i$ and set $A_{i j}=1$ for the sampled values of $j$; and then (c) set the remaining values of $A_{i j}$ to 0 .

Step 0 Sample the uniformity failure times as $\log \tilde{y}_{i}(\mathbf{0}) \sim \mathcal{N}\left(\mu, \sigma^{2}\right)$, where $\left(\mu, \sigma^{2}\right)=\left(4.5,0.25^{2}\right)$.
Step 1 Randomly draw an observed treatment assignment $\mathbf{Z}$ from $\Omega$. Determine the failure time for individual $i$ with observed treatment $\left(Z_{i}, G_{i}\right)$ by $\tilde{Y}_{i}=\tilde{y}_{i}(\mathbf{0}) \exp \left(\delta^{\dagger} Z_{i}+\tau^{\dagger} G_{i}\right)$ for $\left(\delta^{\dagger}, \tau^{\dagger}\right)=(0.7,2.8)$. The values of $\left(\delta^{\dagger}, \tau^{\dagger}\right)$ are chosen so that for $G_{i}>0.25$, the magnitude of the spillover effect is greater than the direct effect, that is, $\tau^{\dagger} G_{i}>\delta^{\dagger}$. The censoring times are then drawn from distributions that depend on treatment. First the dropout times $\tilde{C}_{i}$ are randomly drawn from a lognormal distribution $\log \tilde{C}_{i} \sim \mathcal{N}\left(\mu+\tau^{\dagger} G_{i}, \omega^{2}\right)$, where $\omega^{2}=1-0.25^{2}$. The administrative censoring time is defined as $C_{i}^{\prime}=\exp \left(\mu+2 \sigma+\tau^{\dagger}\right)$. If $Z_{i}=1$, set the censoring time to $C_{i}=\min \left\{C_{i}^{\prime}, \tilde{C}_{i}\right\}$; otherwise, assume there is no dropout and $C_{i}=k C_{i}^{\prime}$ for some specified proportion $k$. Determine the observed outcomes $Y_{i}$ and failure indictors $D_{i}$ as defined above.

Step 2 Under $H_{0}:\left(\delta_{0}, \tau_{0}\right)=(0.7,2.8)$, determine $y_{i}(\mathbf{0})=Y_{i} \exp \left\{-\left(\delta_{0} Z_{i}+\tau_{0} G_{i}\right)\right\}$. For the dataset $\{\mathbf{y}(\mathbf{0}), \mathbf{D}, \mathbf{Z}\}$, carry out the $\log \mathrm{R}$ and LRaft tests, either holding $\mathbf{D}$ fixed over reassignments, or using the proposed method in Section 3.2. The $p$ values $\mathrm{pv}^{C}\left(\delta_{0}, \tau_{0}\right)$ are calculated with $C=10000$.

Step 0 was carried out once, then steps 1 and 2 repeated 2000 times each for $k=1, m=124$.

The empirical cumulative distribution functions (ECDFs) of the LogR and LRaft $p$ values holding $\mathbf{D}$ fixed over reassignments are plotted in the left panel of Figure 1. Neither test controlled the nominal type I error rate in general, with both ECDFs above the diagonal indicating inflated rejection rates of $H_{0}$ above the nominal size. While the empirical type I error rate of the LogR test was below the nominal rate at certain significance levels, this is not guaranteed to be the case in general.
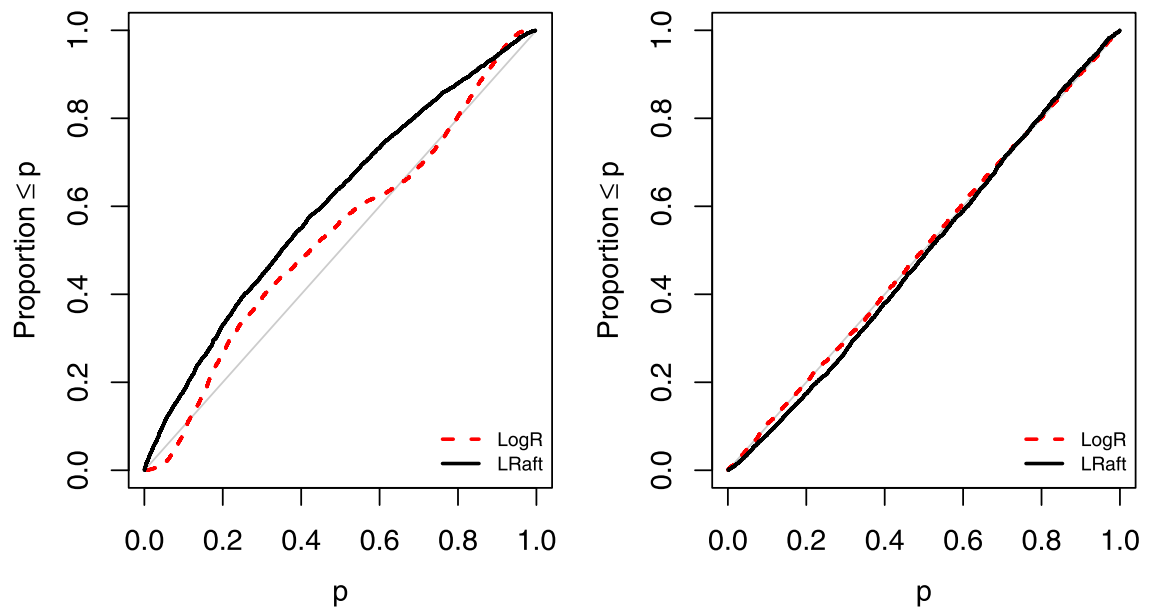

FIG URE 1 Empirical cumulative distributions of $p$ values for the different test procedures described in Section 3.2. For the procedure corresponding to the left panel, the failure indicators $D_{i}$ are held fixed over reassignments. In contrast, the proposed method corresponding to the right panel allows for the set of censored individuals to vary over reassignments [This figure appears in color in the electronic version of this article, and any mention of color refers to that version] 
The empirical results using the proposed method in Section 3.2 are shown in the right panel of Figure 1. The LogR and LRaft tests both had type I error rates that approximately equal the nominal size for all significance levels $\alpha$, with both ECDFs lying approximately on the diagonal. Similar results for other values of $k$ and $m$ are shown in Figures S1 and S2. The proposed method was further evaluated using a different (symmetric) interference structure that was generated as a linear preferential attachment network following Jagadeesan et al. (2017). Settings where the uniformity failure times were correlated between individuals and were correlated with censoring times were also considered. The details of these studies (32 different simulation settings) are given in Supporting Information Appendix A. The results, displayed in Figures S1 to S8, demonstrate that the proposed method controlled the type I error at approximately the nominal level over a variety of scenarios.

Additional simulation studies were conducted to compare the power of the LRaft and LogR tests. The details of these studies are described in Supporting Information Appendix B. The results displayed in Figure 2 correspond to testing the null hypotheses $H_{0}:\left(\delta_{0}, \tau_{0}\right)=$ $(0.6,2.8)$ (left panel) and $H_{0}:\left(\delta_{0}, \tau_{0}\right)=(0.7,3.2)$ (right panel) when the true data generating parameter values were $(\delta, \tau)=(0.7,2.8)$. Power using the LRaft and $\operatorname{LogR}$ tests was similar for $\left(\delta_{0}, \tau_{0}\right)=(0.6,2.8)$, whereas for $\left(\delta_{0}, \tau_{0}\right)=(0.7,3.2)$ the LRaft test was more powerful with $\log \mathrm{R}$ having power approximately equal to the nominal significance level. The observed lack of power of $\log R$ to detect spillover effects different from that posited under the null aligns with intuition since this statistic only compares (censored) uniformity trial outcomes between treated and untreated individuals, with no attempt to account for the proportion (or number) of treated neighbors. The results for other assumed values of $\left(\delta_{0}, \tau_{0}\right)$, as well as empirical coverage of the LRaft and
LogR 95\% confidence sets, are provided in Supporting Information Appendix B.

In summary, the results from these simulation studies indicate that the randomization test procedure in Section 3.2 controls the type I error (empirically) over a range of settings, and the LRaft test tends to be as or more powerful than the LogR test. Moreover, the $\log R$ test can lack power to detect spillover effects and thus is not recommended in practice when assuming the additive causal model.

\section{APPLICATION TO RANDOMIZED TRIAL OF CHOLERA VACCINE}

In this section, the methods described above are utilized to assess the effects of cholera vaccination in a placebocontrolled individually randomized trial in Matlab, Bangladesh (Ali et al., 2005). In prior analyses of these data, Ali et al. (2005) found a negative association between an individual's risk of cholera infection and the proportion of individuals vaccinated in the area surrounding an individual's residence, suggesting possible interference. Similarly, analysis by Emch et al. (2009) found that the risk of cholera was inversely related with vaccine coverage in environmental networks that were connected via shared ponds. Likewise, Root et al. (2011) concluded that the risk of cholera among placebo recipients was inversely associated with level of vaccine coverage in their social networks. Motivated by these association analyses, Perez-Heydrich et al. (2014) used inverse probability weighted estimators to provide evidence of a significant indirect (spillover) effect of cholera vaccination. However, Perez-Heydrich et al. assumed partial interference based on a spatial clustering of individuals into groups and did not account for right
F I G URE 2 Empirical cumulative distributions of $p$ values from a simulation study described in Supporting Information Appendix B using the proposed method in Section 3.2. The true parameter values used to generate the data were $(\delta, \tau)=(0.7,2.8)$. The left panel corresponds to testing the null $H_{0}:\left(\delta_{0}, \tau_{0}\right)=(0.6,2.8)$, and the right panel corresponds to testing the null $H_{0}:\left(\delta_{0}, \tau_{0}\right)=(0.7,3.2)$ [This figure appears in color in the electronic version of this article, and any mention of color refers to that version] $\delta_{0}=0.6, \tau_{0}=2.8$

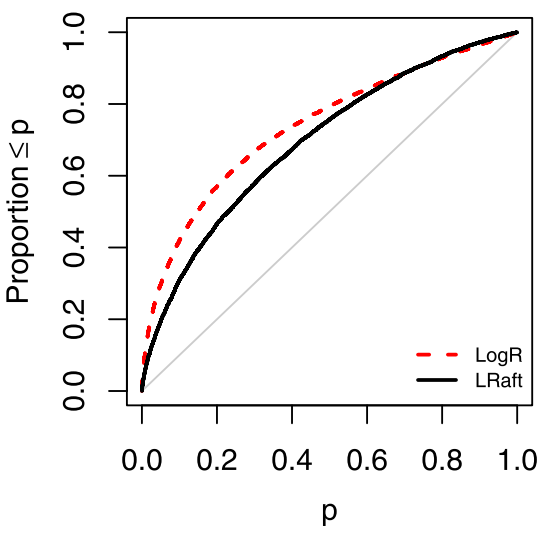

$\delta_{0}=0.7, \tau_{0}=3.2$

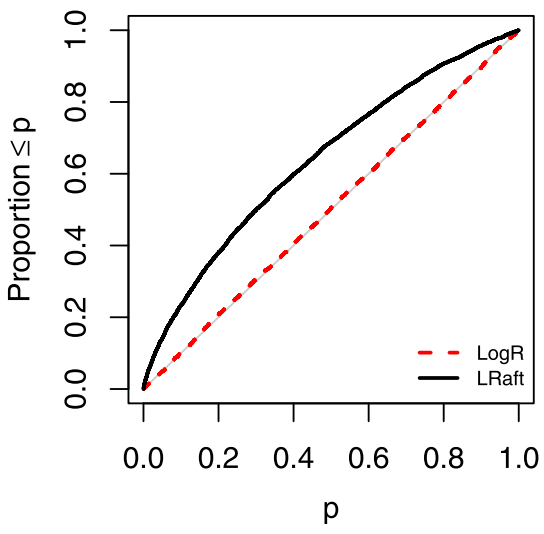


censoring. Misspecification of the interference structure and failure to account for right censoring may bias results. The analysis below considers other possible interference structures and allows for right censoring.

All children aged 2 to 15 years and females over 15 years in the Matlab research site of the International Centre for Diarrheal Disease Research, Bangladesh, were individually assigned randomly to one of three possible treatments: B subunit killed whole-cell oral cholera vaccine; killed whole-cell-only oral cholera vaccine; or Escherichia coli K12 placebo. Recipients of either vaccine were grouped together for analysis as the vaccines were identical in cellular composition and similar in protective efficacy in previous analyses. Denote $Z=0$ for those assigned to placebo, and $Z=1$ for those assigned to either vaccine. Individuals were only included in the analysis if they had completely ingested an initial dose and had completely or almost completely ingested at least one additional dose. There were a total of $n=72965$ individuals in the randomized trial subpopulation for analysis, with $m=48660$ assigned to vaccine and $n-m=24305$ to placebo. The primary outcome for analysis was the (failure) time in days from the 14th day after the vaccination regimen was completed (end of the immunogenic window; Clemens et al., 1988), until a patient was diagnosed with cholera following presentation for treatment of diarrhea. Failure times for many trial participants were right-censored either due to outmigration from the field trial area or death prior to the end of the study, or administrative censoring at the end of the study on 1 June 1986.

\subsection{Interference specifications}

The vaccine trial is analyzed using one of three different specifications of interference in turn. Person-to-person transmission of cholera often takes place within the same bari, that is, geographically clustered households of patrilineally related individuals. Therefore, for all three specifications, an individual's interference set includes all other individuals residing in the same bari. In other words, all individuals $i, j$ residing in the same bari have $A_{i j}=1$. There are 6423 geographically discrete baris with each individual residing in exactly one bari. Three different specifications are posited regarding how an individual's interference set may also include individuals in different baris.

The first specification follows the same approach in Perez-Heydrich et al. (2014). Baris are partitioned into "neighborhoods" according to a single linkage agglomerative clustering method. No interference is assumed between individuals in different neighborhoods and no additional assumptions are imposed regarding the interference structure. That is, partial interference is assumed under this specification. The average number of individuals in each interference set is 419 with an interquartile range (IQR) of 120 to 631 .

Ali et al. (2005) found an association between the cholera risk for a placebo recipient and the vaccine coverage among individuals living within a $500 \mathrm{~m}$ radius of the placebo recipient. Following Ali et al., the second specification of the individual interference sets assumes an individual's potential outcomes may possibly depend on those living in a different bari within a $500 \mathrm{~m}$ radius of the bari $\mathrm{s} / \mathrm{he}$ resided in. This specification does not assume partial interference. The average number of individuals in each interference set under this specification is 499 (IQR 339-626). Baris in the same neighborhood under the first specification may be more than $500 \mathrm{~m}$ apart, for example, in sparsely populated regions; conversely, baris in different (possibly adjacent) neighborhoods may be less than $500 \mathrm{~m}$ apart. Hence, $A_{i j}=1$ under either specification does not imply that $A_{i j}=1$ under the other specification.

The previous two specifications assume a local interference structure based on geographical location of individuals' households. Following Root et al. (2011), the third interference structure is defined according to a kinship-based social network between baris. The Matlab Demographic Surveillance System recorded the exact dates and bari of residence over time for each individual. An individual who migrated between two baris, primarily due to kinship relationships such as marriage, created a nondirectional social tie between the baris. The average number of individuals in each interference set under this specification is 162 (IQR 70-225). Submatrices of the interference matrices for 500 selected participants under each of the three specifications ("neighborhood," " $500 \mathrm{~m}$," and "social") are depicted in Figure 3. The interference matrices for all $n=72965$ participants are shown in Figure S11.

The study population also included 44887 individuals who did not participate in the randomized trial, and thus had zero probability of receiving either cholera vaccine. However, most of these individuals also resided in the same baris as those who took part in the trial: 5661 baris contained a mixture of participants and nonparticipants, with a median participation rate of $71 \%$ within a bari. Since the three specified interference sets are defined based on baris, the definition of $G_{i}$ is expanded to include nontrial participants as follows. Let $B_{i}$ be the total number in the study population, regardless of trial participation, who may possibly interfere with person $i$, so that $B_{i} \geqslant A_{i} \geqslant T_{i}$. Denote the proportion of $B_{i}$ who receive treatment as $G_{i}^{*}$, that is, $G_{i}^{*}=T_{i} / B_{i}$. 

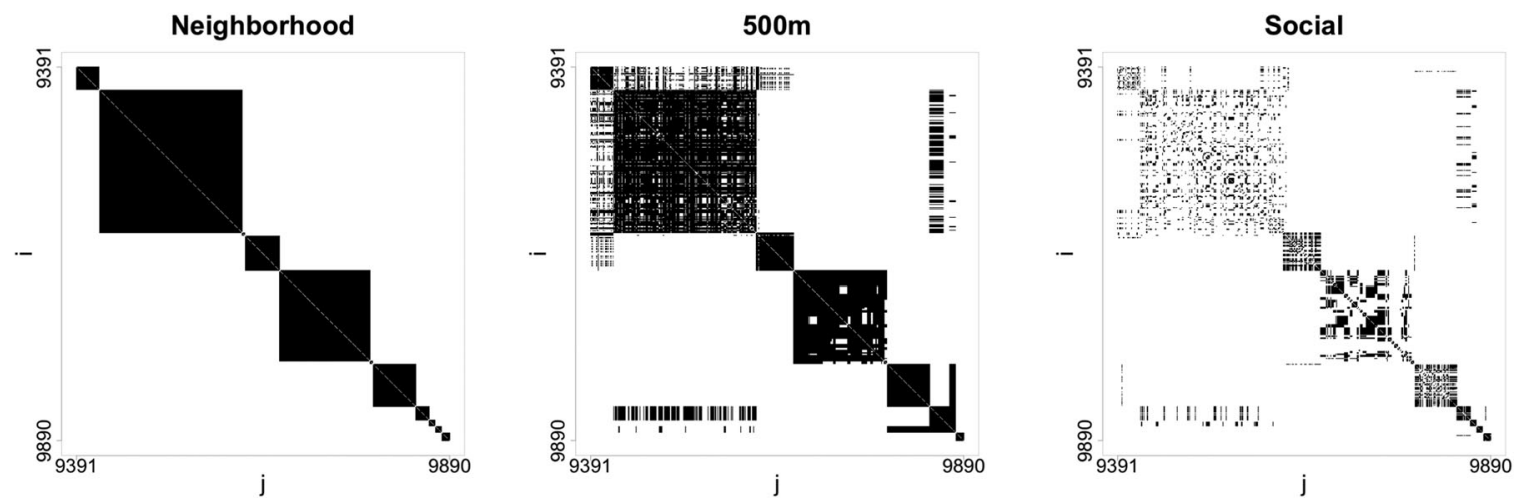

FIG URE 3 Submatrices of the interference matrices for 500 selected participants in the randomized cholera vaccine trial, based on neighborhood (left), $500 \mathrm{~m}$ (center), and social (right) interference specifications
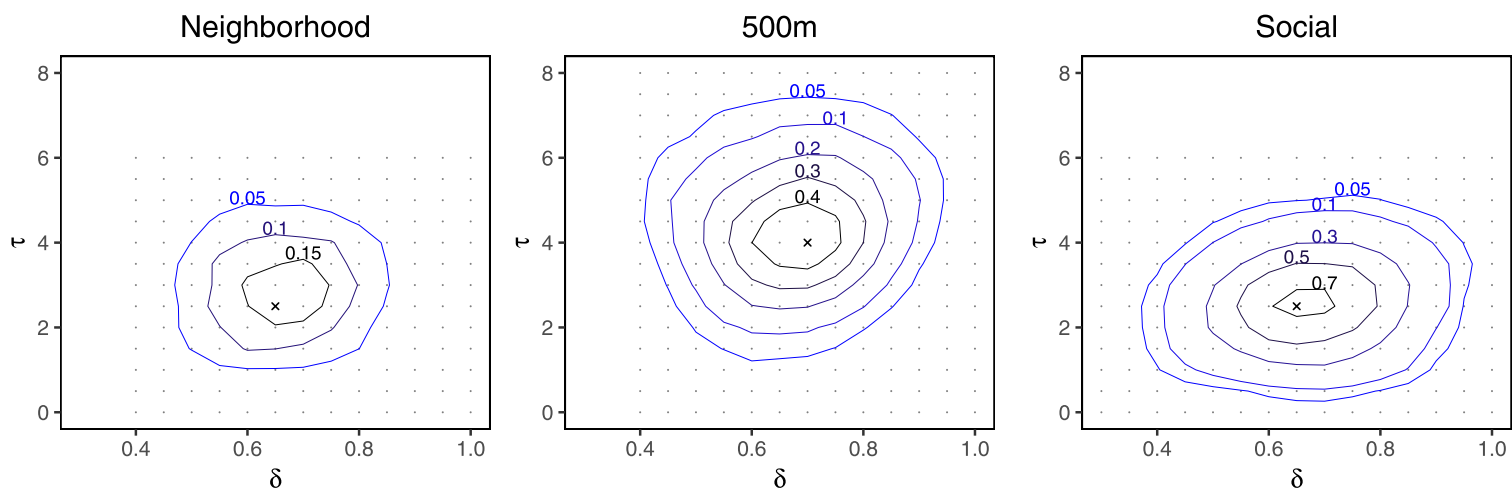

F I G U RE 4 LRaft 95\% confidence sets for $(\delta, \tau)$ under the additive model $\mathcal{F}_{\text {add }}$, and each specified interference matrix (neighborhood, $500 \mathrm{~m}$, or social) for the cholera data. The contours indicate values of $\left(\delta_{0}, \tau_{0}\right)$ yielding the same $p$ values, with darker hues indicating larger $p$ values. The boundaries of the $95 \%$ confidence set are demarcated by the contour lines that indicate $p$ values of at least .05. The point estimate $(\hat{\delta}, \hat{\tau})$ corresponding to the highest $p$ value under each interference structure is indicated by $\mathrm{x}$ [This figure appears in color in the electronic version of this article, and any mention of color refers to that version]

\section{2 | Results}

For each specified interference matrix, confidence sets for $(\delta, \tau)$ were constructed under the causal model $Y_{i}=y_{i}(\mathbf{0}) \exp \left(\delta Z_{i}+\tau G_{i}^{*}\right)$ by conducting hypothesis tests over a discrete grid of values of $\left(\delta_{0}, \tau_{0}\right)$. It was not computationally feasible to enumerate $\Omega$ exactly with $\left(\begin{array}{c}72965 \\ 48660\end{array}\right) \approx 10^{20162}$ possible reassignments, so $p$ values were calculated with $C=4000$ random draws from $\Omega$. The LRaft 95\% confidence sets are plotted in Figure 4, with the contours indicating $\left(\delta_{0}, \tau_{0}\right)$ values yielding the same $p$ values. The boundaries of the $95 \%$ confidence set are demarcated by the contour lines that indicate $p$ values at least as large as .05.

There is evidence that vaccination has an effect on the risk of cholera as the $95 \%$ confidence sets exclude $(\delta, \tau)=(0,0)$ under all three interference specifications. Point estimates of the joint treatment effects, corresponding to values of $\left(\delta_{0}, \tau_{0}\right)$ with the largest $p$ value, are positive, suggesting the effect of the vaccine in reducing the risk of cholera is a combination of protective direct and spillover effects. The direct effect estimates are similar across the three interference specifications, whereas the spillover effect estimate is somewhat higher for the social interference specification. For the $500 \mathrm{~m}$ interference structure, the estimated treatment effect is $(\hat{\delta}, \hat{\tau})=(0.7,4.0)$. We offer two interpretations of $(\hat{\delta}, \hat{\tau})$ under the additive causal model. First, the average time until cholera diagnosis had everyone not received vaccine (ie, the uniformity trial) is estimated to be $\exp (0.7+4.0)=\exp (4.7) \approx 110$ times faster than if everyone had received vaccine (eg, the "blanket coverage" trial). Second, the estimated risk of cholera incidence at 365 days under the uniformity trial would be approximately $2.30 \%$ compared to $0.06 \%$ under the blanket coverage trial, corresponding to a $98 \%$ reduction. The individual parameter estimates also have a straightforward interpretation. For example, holding the proportion of neighbors treated fixed, $\exp (\hat{\delta})=\exp (0.7) \approx 2$ is the estimated ratio of survival times when an individual receives treatment versus control. Similarly, holding individual treatment fixed $\exp (\hat{\tau})=\exp (4.0) \approx 55$ is the 
estimated ratio of survival times when all neighbors are treated compared to no neighbors being treated.

The BFP model was considered unrealistic a priori for this example because there was no plausible scientific rationale for limiting the spillover effect to those who do not receive the vaccine, and to be strictly smaller in magnitude than the direct effect. Nonetheless, for completeness, inference was carried out for parameters under an assumed BFP model. No $p$ values were above .05 , suggesting that the BFP model is a poor fit to the data.

\section{5 | DISCUSSION}

In this paper, we proposed randomization-based methods for assessing the effect of treatment on right-censored outcomes in the presence of general interference. There are several avenues of possible future related research. The adapted $I P_{Z}$ procedure as implemented only allows for unequal censoring based on $Z$. A proportional hazards model may be used in place of the group-specific KM estimators to allow for censoring to differ based on $Z$ and $G$. Building on the empirical results in this paper, future research could examine theoretical properties of the proposed procedures, for example, determine conditions under which type I error rate control is guaranteed. Joint parametric causal models for both the failure times and censoring times in the presence of general interference might also be considered. Since inference is contingent on the choice of interference structure assumed, possible extensions include developing sensitivity analysis methods for assessing robustness to interference structure misspecification. Alternatively, extensions of randomization-based inference approaches that do not require a parametric causal model, such as Sävje et al. (2017), to the setting where outcomes are censored could be considered. Methods such as Jagadeesan et al. (2017) and Athey et al. (2018) that use restricted randomizations to improve statistical power and computational speed might also be considered. While illustrated in this paper using data from an individually randomized trial, the proposed methods can be employed in cluster-randomized trials. Finally, although this paper has focused on two specific causal models, the proposed methods are general and easily extended to other causal models.

\section{ACKNOWLEDGMENTS}

The authors thank Brian Barkley, Sujatro Chakladar, Bradley Saul, the Editor, Associate Editor, and two reviewers for helpful comments. This research was supported by NIH grant R01 AI085073 and by a Gillings Innovation Laboratory award from the UNC Gillings School of Global Public Health. Computational resources and services were provided by the VSC (Flemish Supercomputer Center), funded by the Research Foundation Flanders (FWO) and the Flemish Government-Department EWI. The content is solely the responsibility of the authors and does not represent the official views of the National Institutes of Health.

\section{REFERENCES}

Ali, M., Emch, M., von Seidlein, L., Yunus, M., Sack, D.A., Rao, M. et al. (2005) Herd immunity conferred by killed oral cholera vaccines in Bangladesh: a reanalysis. The Lancet, 366, 44-49.

Athey, S., Eckles, D. and Imbens, G.W. (2018) Exact p-values for network interference. Journal of the American Statistical Association, 113, 230-240.

Bowers, J., Fredrickson, M.M. and Aronow, P.M. (2016) Research note: a more powerful test statistic for reasoning about interference between units. Political Analysis, 24, 395-403.

Bowers, J., Fredrickson, M.M. and Panagopoulos, C. (2012) Reasoning about interference between units: a general framework. Political Analysis, 21, 97-124.

Clemens, J.D., Harris, J.R., Sack, D.A., Chakraborty, J., Ahmed, F., Stanton, B.F. et al. (1988) Field trial of oral cholera vaccines in Bangladesh: results of one year of follow-up. Journal of Infectious Diseases, 158, 60-69.

Collett, D. (2003) Modelling Survival Data in Medical Research, 2nd edition. New York: Chapman \& Hall/CRC.

Emch, M., Ali, M., Root, E.D. and Yunus, M. (2009) Spatial and environmental connectivity analysis in a cholera vaccine trial. Social Science and Medicine, 68, 631-637.

Halloran, M.E. and Hudgens, M.G. (2016) Dependent happenings: a recent methodological review. Current Epidemiology Reports, 3, 297-305.

Hudgens, M.G. and Halloran, M.E. (2008) Toward causal inference with interference. Journal of the American Statistical Association, 103, 832-842.

Jagadeesan, R., Pillai, N. and Volfovsky, A. (2017) Designs for estimating the treatment effect in networks with interference. Available at: https://arxiv.org/abs/1705.08524

Lehmann, E.L. and Romano, J.P. (2005) Testing Statistical Hypotheses. Springer Texts in Statistics, 3rd edition. New York: Springer.

Ogburn, E.L., Sofrygin, O., Diaz, I. and van der Laan, M.J. (2017) Causal inference for social network data. Available at: https:// arxiv.org/abs/1705.08527

Perez-Heydrich, C., Hudgens, M.G., Halloran, M.E., Clemens, J.D., Ali, M. and Emch, M.E. (2014) Assessing effects of cholera vaccination in the presence of interference. Biometrics, 70, 731-741.

Root, E.D., Giebultowicz, S., Ali, M., Yunus, M. and Emch, M. (2011) The role of vaccine coverage within social networks in cholera vaccine efficacy. PLOS One, 6. e22971.

Rosenbaum, P.R. (2002) Observational Studies. New York: Springer. Rosenbaum, P.R. (2007) Interference between units in randomized experiments. Journal of the American Statistical Association, 102, 191-200.

Sävje, F., Aronow, P.M. and Hudgens, M.G. (2017) Average treatment effects in the presence of unknown interference. Available at: https://arxiv.org/abs/1711.06399 
Sobel, M.E. (2006) What do randomized studies of housing mobility demonstrate? Causal inference in the face of interference. Journal of the American Statistical Association, 101, 1398-1407.

Sussman, D.L. and Airoldi, E.M. (2017) Elements of estimation theory for causal effects in the presence of network interference. Available at: https://arxiv.org/abs/1702.03578

Wang, R., Lagakos, S.W. and Gray, R.J. (2010) Testing and interval estimation for two-sample survival comparisons with small sample sizes and unequal censoring. Biostatistics, 11, 676-692.

\section{SUPPORTING INFORMATION}

Web Appendices A and B and Web Figures 1-11 referenced in Sections 3.3 and 4.1 are available with this paper at the Biometrics website on Wiley Online Library. The $\mathrm{R}$ code used to implement the proposed methods and to carry out the simulation studies in Section 3.3 and in Web Appendices A and B are available at the following web address: https://github.com/wwloh/General-Interference-Censoring. 